\title{
How did the Beneficiary Groups of Curative Care Expenditure Distribute? Based on SHA 2011: A Case Study in Xinjiang Autonomous Region, China
}

\section{Honghong Feng}

Shihezi University School of Medicine https://orcid.org/0000-0003-0385-6704

Lu Mao

Shihezi University School of Medicine

\section{Xiaoju Li}

Shihezi University School of Medicine

\section{Liwen Zhang}

Shihezi University School of Medicine

Dongsheng Rui ( $\Delta$ 18909934451@qq.com )

Shihezi University School of Medicine https://orcid.org/0000-0001-8701-6600

\section{Research}

Keywords: SHA 2011, beneficiary, curative care expenditure, Xinjiang

Posted Date: May 28th, 2020

DOI: https://doi.org/10.21203/rs.3.rs-30349/v1

License: (c) (i) This work is licensed under a Creative Commons Attribution 4.0 International License. Read Full License 


\section{Abstract \\ Background}

System of Health Accounts 2011 (SHA2011) not only meets the analysis of health policies but also increases the comparability of international health from expenditure. This study analyzes the distribution of beneficiary groups of curative care expenditure (CCE) in Xinjiang based on SHA 2011, and provides references for making health policies.

\section{Methods}

A total of 352 sample health institutions were collected with multistage stratified random sampling method in Xinjiang. According to the accounting framework of the SHA 2011, the CCE distribution, institutional flow and disease distribution of different age groups were analyzed.

\section{Results}

In 2016, the CCE in Xinjiang was 50.05 billion Chinese Yuan (CNY), accounting for $70.18 \%$ of current health expenditure (CHE), $6.66 \%$ of GDP, and per capita CCE was 2366.56 CNY. People aged over 65 was the consumed major CCE, different diseases treatment costs have a different distribution in the age groups. Three types of diseases with the highest CCE were circulatory, respiratory and digestive system diseases. All of the beneficiary expenditure mainly occurred in hospitals, the proportion of primary health care institutions was relatively small. Family health expenditure accounts for a relatively high proportion, especially children aged 14 years old and below.

\section{Conclusion}

This study used SHA2011 to capture large data and analyzed from the newly added beneficiary dimension. The population had a heavy burden of medical financing, the allocation of medical resources was unreasonable, and the scale of primary medical institutions needs to be reasonably expanded. The financing plan for CCE needs to be optimized to decrease the medical burden of disease.

\section{Background}

To analyze the increasing expenditure of health, international organizations have formulated the "System of Health Accounts 2011" (SHA2011), which not only meets the analysis of health policies but also increases the comparability of international health from expenditure [1]. Compared with SHA1.0, SHA2011 added the dimension of health service beneficiaries, met the needs of evolving health care systems[2]. 
When the first draft of SHA2011 was formed in 2010, China National Health Accounts (NHA) team was invited by WHO to participate in the experimental research of SHA2011. China NHA team conducted an experimental study in Tianjin in 2010 using the SHA2011, which verified its feasibility and provided valuable experience for final improvement of SHA2011[3].

The implementation process of SHA2011 in China started first at several pilot provinces, and then fully carried out the accounting work of SHA2011 nationwide. From 2013 to 2014 year, a pilot project of accounting based on SHA2011 was carried out in Gansu, Sichuan, Jilin and Tianjin provinces.

Subsequently, the health expenditure accounting was extended to 8 other provinces, and the project was further mature in China. In July 2016, China National Health Development Research Center organized a national SHA2011's method training conference in Beijing, which required all regions of the country to fully carry out SHA2011 accounting. Xinjiang officially launched SHA2011 accounting in 2016.

This study is based on SHA2011 beneficiary dimension having analyzed the distribution of curative care expenditure (CCE) in Xinjiang in 2016. It has described the matrix relationship between different age groups and diseases, institutional flow, financing scheme. It also helps to understand the status of health resource consumption in different populations and determine the focus of the key populations and diseases in Xinjiang. Therefore, this study uses Xinjiang health data as an example to explore the distribution of beneficiaries for CCE, and provide data support for improving health-related policies formulation.

\section{Methods}

\section{Data Source}

The basic data of this study derived from official statistic data of Xinjiang in 2016, such as "Health Statistics Yearbook", "Health Financial Annual Report" and "Government Health Input Testing System". The case database came from 352 sample health institutions in Xinjiang.

\section{Data Sample}

This study was based on the SHA 2011 and used a multistage stratified random sampling method. The first stage was selected five areas as sample areas, according to the economic development status and medical services level, then we chose Urumqi, Kashgar, lli areas, Bayinguole Mongolian Autonomous Prefecture and Hami. Next stage, basing on the comprehensive capabilities, working foundation and information construction of each medical institution, 1 city and 2 counties were selected within each selected prefecture. Finally, five communities or townships were selected as samples from each of the selected cities and counties.

In total, 352 health institutions were selected as samples. The data of outpatient and inpatient cases collected from the sample survey institutions after cleaning up. The data meeting the requirements was 
about 4.3 million. This kind of data was mainly used to calculate the allocation parameters of each dimension.

\section{Statistical method}

CCE in our country include treatment, rehabilitation and long-term care services expenditure[4]. China's health service institutions provide treatment, rehabilitation and long-term care services. The three services are intertwined and difficult to separate.

Hospitals, public health institutions, and basic medical institutions have the same method of calculating CCE. To calculate CCE, we used the following formula:

$$
\mathrm{C}_{\mathrm{CCE}}=\sum_{\mathrm{K}=1}\left(\mathrm{C}_{\mathrm{KINC}}+\mathrm{C}_{\mathrm{KALL}}\right)
$$

In this formula $\mathrm{C}_{\mathrm{KINC}}$ represents the curative income, $\mathrm{C}_{\mathrm{KALL}}$ represents the basic expenditure allowance, $\mathrm{K}$ represents different medical institutions.

The formula for calculating curative income is as follows:

$$
\begin{gathered}
\mathrm{C}_{\mathrm{INC}}=\mathrm{C}_{\mathrm{TINC}}\left(1-\frac{\alpha_{\mathrm{p}}}{\alpha}\right) \\
\mathrm{C}_{\mathrm{INC}}^{\prime}=\sum_{\mathrm{i}}^{\mathrm{n}}\left(\mathrm{C}_{\mathrm{INC}} \times \frac{\alpha_{\mathrm{i}}}{\alpha-\alpha_{\mathrm{p}}}\right)
\end{gathered}
$$

Above all of the formulas, $\mathrm{C}_{\mathrm{TINC}}$ represents the actual total income from Health Statistics Yearbook and Health Financial Annual Report, a represents the total curative income of sample, $a_{p}$ represents total curative income of sample preventive service. $C_{\text {INC }}^{\prime}$ represents the actual curative income, $a_{i}$ represents curative income in sample patient.

The formula for calculating basic expenditure allowance is as follows:

$$
C_{\mathrm{ALL}}^{\prime}=\sum_{\mathrm{i}}^{\mathrm{m}}\left[\left(\mathrm{C}_{\mathrm{TALL}}-\mathrm{C}_{\mathrm{PALL}}\right) \times \frac{\alpha_{\mathrm{i}}}{\alpha-\alpha_{\mathrm{p}}}\right]
$$

$\mathrm{C}_{\mathrm{ALL}}^{\prime}$ represents the total actual basic expenditure allowance. $\mathrm{C}_{\mathrm{TALL}}$ represents the basic expenditure allowance from Health Statistics Yearbook and Health Financial Annual Report, when $a_{i}$ represents curative income in sample patient. $\mathrm{C}_{\text {TALL }}$ represents the basic expenditure allowance of preventive service. 
STATA12.0 and SPSS20.0 software were used for the analyses.

\section{Results}

\section{Basic information about CCE of different age groups in Xinjiang in 2016}

In 2016, the CCE in Xinjiang was 50.05 billion Chinese Yuan(CNY), accounting for $70.18 \%$ of current health expenditure, and accounting for $6.66 \%$ of GDP. And per capita CCE was 2366.56 CNY. The main CCE was for hospitalization. The hospitalization cost was 23.136 billion CNY, accounting for $46.23 \%$ of the CCE; the outpatient cost was 11.986 billion CNY, accounting for $23.95 \%$ of the CCE.

The results of CCE for people of different ages showed that the CCE decreased with age increasing from 0 to 14 years group. From 15 to 54 years group, the CCE continued to increase with age, reaching the highest expenditure in the 50-54 age group. Over the 55 years group, the CCE had declined with age increasing. Different ages consumed different degrees of health resources, so expenditure also changes with age (Table 1$)$.

Comparing the proportion of the resident population with the proportion of CCE in each age group, it was found that the proportion of CCE for the people under 64 was lower than the proportion of the population, especially for those under 14 years old. The proportion of CCE for people over 65 was significantly higher than the proportion of their population, indicating that the elderly population consumes more medical resources (Fig. 1).

Table 1. The cost of treatment in Xinjiang by age group in 2016 


\begin{tabular}{|c|c|c|c|c|c|c|}
\hline Age & $\begin{array}{c}\text { Total/ } \\
\text { Billion CNY }\end{array}$ & Ratio/\% & $\begin{array}{l}\text { Outpatient/ } \\
\text { billion CNY }\end{array}$ & Ratio/\% & $\begin{array}{l}\text { Intpatient/ } \\
\text { billion CNY }\end{array}$ & Ratio/\% \\
\hline $0 \sim$ & 2.46 & 4.92 & 0.75 & 4.42 & 1.71 & 5.18 \\
\hline $5 \sim$ & 1.32 & 2.64 & 0.78 & 4.55 & 0.55 & 1.66 \\
\hline $10 \sim$ & 0.80 & 1.60 & 0.44 & 2.55 & 0.37 & 1.11 \\
\hline $15 \sim$ & 0.97 & 1.97 & 0.47 & 2.73 & 0.52 & 1.58 \\
\hline $20 \sim$ & 1.52 & 3.04 & 0.68 & 4.00 & 0.84 & 2.53 \\
\hline $25 \sim$ & 2.69 & 5.38 & 1.18 & 6.90 & 1.51 & 4.59 \\
\hline $30 \sim$ & 2.73 & 5.46 & 1.18 & 6.89 & 1.56 & 4.72 \\
\hline $35 \sim$ & 2.75 & 5.50 & 1.10 & 6.45 & 1.65 & 5.00 \\
\hline $40 \sim$ & 3.98 & 7.95 & 1.46 & 8.56 & 2.52 & 7.63 \\
\hline $45 \sim$ & 4.86 & 9.71 & 1.68 & 9.81 & 3.19 & 9.66 \\
\hline $50 \sim$ & 5.09 & 10.18 & 1.71 & 10.02 & 3.38 & 10.26 \\
\hline $55 \sim$ & 4.11 & 8.21 & 1.24 & 7.28 & 2.87 & 8.69 \\
\hline $60 \sim$ & 4.37 & 8.73 & 12.55 & 7.35 & 3.11 & 9.44 \\
\hline $65 \sim$ & 3.74 & 7.47 & 0.96 & 5.61 & 2.78 & 8.44 \\
\hline $70 \sim$ & 3.28 & 6.55 & 0.83 & 4.85 & 2.45 & 7.43 \\
\hline $75 \sim$ & 2.94 & 5.88 & 0.73 & 4.29 & 2.21 & 6.70 \\
\hline $80 \sim$ & 1.54 & 3.07 & 0.41 & 2.40 & 1.13 & 3.42 \\
\hline $85 \sim$ & 0.66 & 1.33 & 0.18 & 1.03 & 0.49 & 1.48 \\
\hline $90 \sim$ & 0.17 & 0.34 & 0.05 & 0.28 & 0.12 & 0.37 \\
\hline$\geq 95$ & 0.03 & 0.07 & 0.01 & 0.04 & 0.03 & 0.08 \\
\hline Total & 50.05 & 100.00 & 17.08 & 100.00 & 32.97 & 100.00 \\
\hline
\end{tabular}

\section{Disease distribution of CCE for different age groups}

According to the classification criteria of the main chapter of the ICD-10, the top five CCE in Xinjiang were circulatory system disease, respiratory system disease, digestive system disease, urogenital system disease, muscular and connective tissue diseases totaled 28.646 billion CNY. The total expenditure of the top five diseases accounted for $57.24 \%$. The composition of diseases that consumes medical resources in different age groups is different. The highest CCE for respiratory diseases in people under 14 was $39.71 \%$, followed by symptoms, signs and clinical and laboratory abnormalities and digestive diseases. From 15 to 64 years group the highest CCE for circulatory diseases was $16.06 \%$, followed by respiratory diseases, digestive diseases and urogenital diseases. The highest CCE for people over 65 years group 
was circulatory diseases accounted for $34.55 \%$, followed by respiratory diseases and tumors accounted for a relatively high proportion (Table 2 ).

\section{Medical institutions distribution of CCE for different age groups}

Among the analysis of the provision of medical service providers, the CCE in 2016 mainly occurred in hospitals, with a total of 38.245 billion CNY, accounting for $76.42 \%$ of CCE, of which general hospitals were the main, followed by traditional Chinese medical hospitals and specialized hospitals. The treatment expenses incurred in primary health care institutions was 10.953 billion CNY, accounting for $21.89 \%$ of treatment expenses, and the proportion incurred in outpatient institutions and maternal and child health care was relatively small. Medical service providers differ in the cost of treatment for people of different ages.

Table 2. Distribution of diseases by cost of treatment at different ages $\square \% \square$ 


\begin{tabular}{|c|c|c|c|c|}
\hline ICD-10 & $\leq 14$ & $15 \sim$ & $\geq 65$ & Total \\
\hline Circulatory system diseases & 1.04 & 16.06 & 34.55 & 19.11 \\
\hline Respiratory system diseases & 39.71 & 10.19 & 15.32 & 14.92 \\
\hline Digestive system diseases & 7.90 & 9.82 & 7.37 & 9.06 \\
\hline Urogenital system diseases & 3.07 & 9.08 & 4.34 & 7.33 \\
\hline Muscular and connective tissue diseases & 1.38 & 7.81 & 6.55 & 6.83 \\
\hline Injury, poisoning and external causes & 5.37 & 7.35 & 3.59 & 6.17 \\
\hline Endocrine, nutritional and metabolic disease & 2.86 & 5.93 & 6.74 & 5.82 \\
\hline Tumor & 1.20 & 5.37 & 8.43 & 5.68 \\
\hline Symptoms, signs, clinical and laboratory abnormalities & 9.04 & 6.36 & 2.19 & 5.51 \\
\hline Mental and behavior disorders & 0.69 & 4.28 & 1.78 & 3.28 \\
\hline Factors influencing health status & 1.54 & 4.26 & 1.21 & 3.24 \\
\hline Infectious diseases and parasitic diseases & 5.54 & 2.62 & 1.55 & 2.59 \\
\hline Nervous system diseases & 3.69 & 2.14 & 1.89 & 2.19 \\
\hline Pregnancy, childbirth and puerperium diseases & 0.04 & 3.16 & 0.00 & 2.06 \\
\hline Skin and subcutaneous tissue disease & 2.31 & 2.27 & 0.83 & 1.89 \\
\hline Eye diseases & 0.47 & 1.13 & 2.58 & 1.41 \\
\hline Blood diseases & 2.01 & 0.77 & 0.51 & 0.80 \\
\hline Perinatal diseases & 6.73 & 0.15 & 0.00 & 0.70 \\
\hline Congenital anomalies and chromosomal abnormalities & 3.85 & 0.49 & 0.14 & 0.70 \\
\hline Ear diseases & 1.31 & 0.61 & 0.38 & 0.61 \\
\hline Death & 0.24 & 0.15 & 0.06 & 0.13 \\
\hline Total & 100.00 & 100.00 & 100.00 & 100.00 \\
\hline
\end{tabular}

The cost of treatment for people over 65 years of age was mainly in hospitals, accounting for $82.43 \%$, while the proportion in primary health care institutions was small. The cost of treatment for people under 14 years of age was mainly in general hospitals. Followed by primary health care institutions, and then specialized hospitals. Among different types of hospitals, it was found that the treatment cost of those under 14 years of age was higher in specialist hospitals, while the treatment costs of older people were higher in traditional Chinese medical hospitals (Table 3 ).

Table 3. Distribution of medical service providers by age (\%ם 


\begin{tabular}{lcccc}
\hline \multicolumn{1}{c}{ Medical service providers } & $\leq 14$ & $15 \sim$ & $\geq 65$ & Total \\
\hline Hospitals: & 66.18 & 75.59 & 82.43 & 76.42 \\
\multicolumn{1}{c}{ general hospital } & 43.68 & 59.06 & 63.56 & 58.76 \\
traditional Chinese medical hospital & 5.18 & 11.51 & 14.93 & 11.78 \\
& 17.32 & 5.02 & 3.95 & 5.88 \\
$\quad$ specialized hospital & 0.00 & 0.03 & 0.00 & 0.02 \\
Maternal and child care centers & 2.46 & 1.92 & 0.69 & 1.67 \\
Outpatient service institutions & & & & \\
Primary health agents & 31.36 & 22.45 & 16.87 & 21.89 \\
\hline Total & 100.00 & 100.00 & 100.00 & 100.00 \\
\hline
\end{tabular}

\section{Financing distribution of CCE for different age groups}

Among the CCE of Xinjiang, in 2016, the total funding of public financing scheme was 31.368 billion CNY, accounting for $62.68 \%$ of CCE, of which government programs accounted for $28.13 \%$, and social medical insurance programs accounted for $34.55 \%$; followed by household health expenditures of 17.097 billion CNY, accounting for $34.16 \%$; voluntary funding schemes account for $3.16 \%$ of CCE. Funding schemes for different ages are also different. The main source of funding for people under the age of 14 is family household health expenditure accounting for $47.82 \%$, followed by government funding and social health insurance; while those over the age of 15 are mainly social health insurance, followed by slightly different sources of funding. The 15-64 year-old group is the government financing scheme, and over the 65-yearold group is the family health expenditure (Table 4).

Table 4. Distribution of funding options for treatment costs for different age groups (\%)

\begin{tabular}{lcccc}
\hline Health financing & $\leq 14$ & $15-65$ & $65 \geq$ & Total \\
\hline Public financing scheme & 49.72 & 70.17 & 68.15 & 62.68 \\
Government financing scheme & 34.40 & 27.65 & 22.34 & 28.13 \\
Social health insurance & 15.32 & 42.52 & 45.81 & 34.55 \\
Voluntary financing scheme & 2.47 & 3.47 & 3.53 & 3.16 \\
Family health expenditure & 47.82 & 26.36 & 28.32 & 34.16 \\
Total & 100.00 & 100.00 & 100.00 & 100.00 \\
\hline
\end{tabular}

\section{Discussions}

Breakdown of treatment costs by age can reflect the burden of health costs of different age groups. It has described the matrix relationship between different age groups and diseases, institutional flow, financing 
scheme, can further determine the economic burden of major diseases in different age groups, and the medical treatment flow and financing risk protection status.

Intervention in key groups and diseases that consume treatment cost to reduce CCE. For controlling the growth of health expenditure, we should pay more attention to the above 65 years old and give more policy preference $[5,6]$. China has entered the aging stage, we should strengthen the preventive medicine and health services for the elderly, so as to achieve the control of medical and health costs for the elderly[7]. For people over 65 years old, we should reduce the cost burden of diseases by three-level prevention, standardized medication, and reasonable treatment, strengthen the daily management of chronic diseases and cancer screening, and effectively control the medical cost of the elderly[8, 9]. In different age groups, the highest cost of respiratory disease treatment was the people under 14 years old. At the same time of protecting children's health, to better control the health costs of children growth. With the implementation of the two-child policy, we need to pay more attention to children's health, and the cost of disease treatment will continue to increase [10]. Combining with centralized characteristics of children's treatment costs and diseases distribution, it is necessary to do a good job in the health education for children's parents and children. To raise the level of parental prevention of children's common diseases and frequently-occurring diseases, especially respiratory system diseases. For the young and middle-aged people aged 15-64, we should strengthen the screening of diseases, advocate a healthy lifestyle, to achieve the increase of disease prevention and control costs[11].

Reasonable allocation of medical resources, different age groups of CCE flow is differently. The CCE mainly flows to the hospital, which shows that there are unreasonable problems in the flow of medical treatment and the allocation of medical funds among medical institutions at all levels. Hospitals bear too much pressure on human resources and need to develop and improve the medical mechanism $[12,13]$. According to the priority of the disease and the difficulty of treatment, different levels of institutions undertake different treatment tasks. Adjust the scale of primary medical and health institutions, strengthen the infrastructure construction and medical staff allocation, implement the contracted services of community general practitioners, and share the treatment pressure[14]. This study found that under-14-year-old is the main target of primary medical institutions and specialized hospital, while over65-year-old people prefer to go to traditional Chinese medicine hospital. Overall, it shows that the allocation of CCE in Xinjiang is unreasonable. To improve the medical level of primary medical institutions, focus on the diagnosis and treatment of common diseases and frequently occurring diseases, to achieve the majority of diseases can be treated at the primary medical institutions $[15,16]$. For the middle-aged and elder, chronic disease intervention is the main way to reduce CCE, and primary medical institutions are the main institutions for the prevention and treatment of chronic diseases. Primary medical institution not only disseminates knowledge about the risk factors of chronic diseases, but also provides medicine and health counseling to help them develop good living and working habits.

The burden of CCE is still heavy, and the financing scheme needs to be optimized. There are significant differences in health financing structure among different age groups. Among the groups, the family burden of under-14-year-olds is the heaviest. At present, there are still some obstacles to the coverage and 
reimbursement of medical insurance for children, especially newborns, which leads to a high proportion of children's medical expenses in the family burden. The social medical insurance in the autonomous region is mainly "hospitalization insurance", and the proportion of different types of insurance in social insurance is different. Compared with other age groups, the proportion of hospitalization service utilization for the elderly is relatively high, the proportion of social medical insurance reimbursement in its health expenses is correspondingly high, while the proportion of family burden is relatively low. A reasonable health financing system should focus on public financings such as government and social medical insurance, and control the personal cash health expenditure (OOP) paid by the family to a lower level[17]. If the health financing system relies too much on household health expenditures, it will easily lead to catastrophic expenditure or poverty due to illness [18]. Due to physical reasons, children's resistance is poor and they are more likely to get sick, which not only leads to the loss of children's health but also brings a huge cost of disease treatment. Besides, as a protection group, children need to rely on the support and care of family and society. Therefore, the cost of disease treatment will be directly converted into the economic burden of family and society [10]. So, encouraging the development of children's commercial medical insurance can also enhance security. The coverage of basic medical insurance after the integration of urban and rural residents' medical insurance will be conducive to the stable development and balance of the financing system with public financing as the main body[19, 20]. Encouraging enterprises and individuals to participate in commercial medical insurance, and strengthening the stability of the financing structure to a certain extent.

\section{Conclusion}

This study used SHA2011 to capture large data and analyzed from the newly added beneficiary dimension. The population had a havey burden of medical financing, the allocation of medical resources was unreasonable, and the scale of primary medical institutions needs to be reasonably expanded, the financing scheme for CCE needs to be optimized. Health policy-making department need to optimize relevant policies to decrease the medical burden of disease.

\section{Abbreviations}

\section{CCE}

curative care expenditure

SHA 2011

System of Health Accounts 2011

$\mathrm{NHA}$

China National Health Accounts

\section{Declarations}

\section{Ethics approval and consent to participate}


The study was supported by Health Commission of Xinjiang Uygur Autonomous Region and they claimed that they approved this study. All procedures performed in studies involving human participants were in accordance with the ethical standards of the institutional and national research committee and with the Helsinki declaration and its later amendments or comparable ethical standards.

\section{Consent for publication}

Not Applicable.

\section{Availability of data and materials}

The datasets generated and/or analyzed during the current study are available from the corresponding author on reasonable request.

\section{Competing Interests}

The authors declare that there is no conflict of internet. No competing financial interests exist.

\section{Funding}

The authors received support of Ministry of Education of the People's Republic of China (CN) Project of Humanities and Social Sciences- Xinjiang (No.18XJJCZH001)

\section{Authors' Contributions}

Feng had full access to all of the study and takes responsibility for the integrity of the data and the accuracy of the data analysis.

Concept and design: Rui.

Acquisition, analysis, or interpretation of data: All authors.

Drafting of the manuscript: Feng and Mao.

Helping revise the manuscript: Li, Zhang, Rui.

Statistical analysis: Li, Zhang, Mao.

All authors read and approved the final manuscript.

\section{Acknowledgments}

The authors express thanks to China National Health Development Research Center for their help with SHA 2011 analysis, and we also gratefully acknowledge the support of Health Commission of Xinjiang Uygur Autonomous Region. Thanks for Xinjiang's department of finance, civil affairs and other 
departments to provide basic accounting data, and thanks for the data support provided by the sampled medical and health institutions.

\section{References}

1. OECD, WHO, Eurostat: A System of Health Accounts. 2011.

2. Mueller M, Morgan D. New insights into health financing: First results of the international data collection under the System of Health Accounts 2011 framework. Health Policy. 2017;121:764-9.

3. Zhai T, Zhang Y, Wan Q, Chai P, Wei Q, Wang C, Guo F, Zhao Y. New System of Health Accounts Introduction of System of Health Accounts 2011. Chinese Health Economics 2013;15-17.

4. Zhai T, Zhang Y, Wan Q, Chai P, Guo F, Wang C, Wang X. Methodological Research on China Health Expenditure Estimation Based On SHA 2011. Chinese Health Economics 2015;11-13.

5. Duan W, Zheng A, Mu X, Li M, Liu C, Huang W, Wang X. How great is the medical burden of disease on the aged? Research based on "System of Health Account 2011". Health Qual Life Outcomes. 2017;15:134-4.

6. Jiang Y, Zhao X, Zhao L, Man X, Wang W, Li B, Cheng W. Analysis on the distribution of curative care expenditure for the beneficiaries of treatment services in Beijing: Based on the System of Health Account 2011. Chinese Journal of Health Policy 2018; 7-12.

7. Yang Y, Zheng A, Li M, Duan W, Mu X, Wang X. Medical economic burden of the ageing population: a multistage sampling analysis of $3+\bigotimes 532+\llbracket 517$ cases. The Lancet. 2016;388:79.

8. Huang S, Jia J, Xiong K, Feng L, Zhong Z, Liu Z, Sun Q, Yang L. Analyzing The Treatment Cost Accounting of The Elderly in Sichuan Province Based on The "System of Health Accounts (SHA) 2011". Chinese Health Service Management 2019; 191-193 + 200.

9. Luo J, Li Y, Li J, Wang P, Jian W, Liying Z, Zhang M, Bian S. Analysis of the Status-quo of Service in Primary Health Care Institutions in Tianjin-Based on the Perspective of Regular Health Costs. Health Economics Research 2019; 50-53.

10. Li M, Zheng A, Duan W, Mu X, Liu C, Yang Y, Wang X. How to apply SHA 2011 at a subnational level in China's practical situation: take children health expenditure as an example. J Glob Health. 2018;8:010801.

11. Yang L, Huang $Y$, Sun Q, Wang Y, Wang M, Huang S. Analyzing the Beneficiary Groups of Curative Care Expenditure in Sichuan Based on SHA 2011. Chinese Health Service Management 2017; 41-44.

12. Zang S, Zhan H, Zhou L, Wang X. Research on Current Curative Expenditure among Lung Cancer Patients Based on the "System of Health Accounts 2011": Insights into Influencing Factors. J Cancer. 2019;10:6491-501.

13. Song S, Ma X, Zhang L, Yuan B, Meng Q. Precision targeting for more equitable distribution of health professionals in rural China. Health Policy Plan. 2018;33:821-7.

14. Dong Y, Liu C, Zhou P, Zhu Y, Tang Q, Wang S, Wang X. How Serious is the Economic Burden of Diabetes Mellitus in Hainan Province? A Study Based on "System of Health Account 2011". Diabetes 
Therapy. 2019;10:2251-63.

15. He J, Yin Z, Duan W, Wang Y, Wang X. Factors of hospitalization expenditure of the genitourinary system diseases in the aged based on "System of Health Account 2011" and neural network model. Journal of global health 2018, 8.

16. Ren Y, Yang X, Shen C, Jia X, Zhou Z, Xu T, Cao D. Study on the Distribution of Treatment Costs for Different Age Groups in Shaanxi Province-Based on Regular Health Costs. Health Economics Research 2019:48-50 + 54.

17. Zhan H, Wu Q, Zang S, Zhou LR, Wang X. How were situations of preventive and curative care expenditure for AIDS and medical burden of patients? Research based on "System of Health Accounts 2011". Bmc Public Health 2020, 20.

18. Zheng A, Duan W, Zhang L, Bao X, Mao X, Luo Z, Jin F. How great is current curative expenditure and catastrophic health expenditure among patients with cancer in China? A research based on "System of Health Account 2011". Cancer Med. 2018;7:4036-43.

19. Song S, Yuan B, Zhang L. Increased Inequalities in Health Resource and Access to Health Care in Rural China. 2018, 16.

20. Chen Y, Yin Z, Xie Q. Suggestions to ameliorate the inequity in urban/rural allocation of healthcare resources in China. International Journal for Equity in Health. 2014;13:34.

\section{Figures}

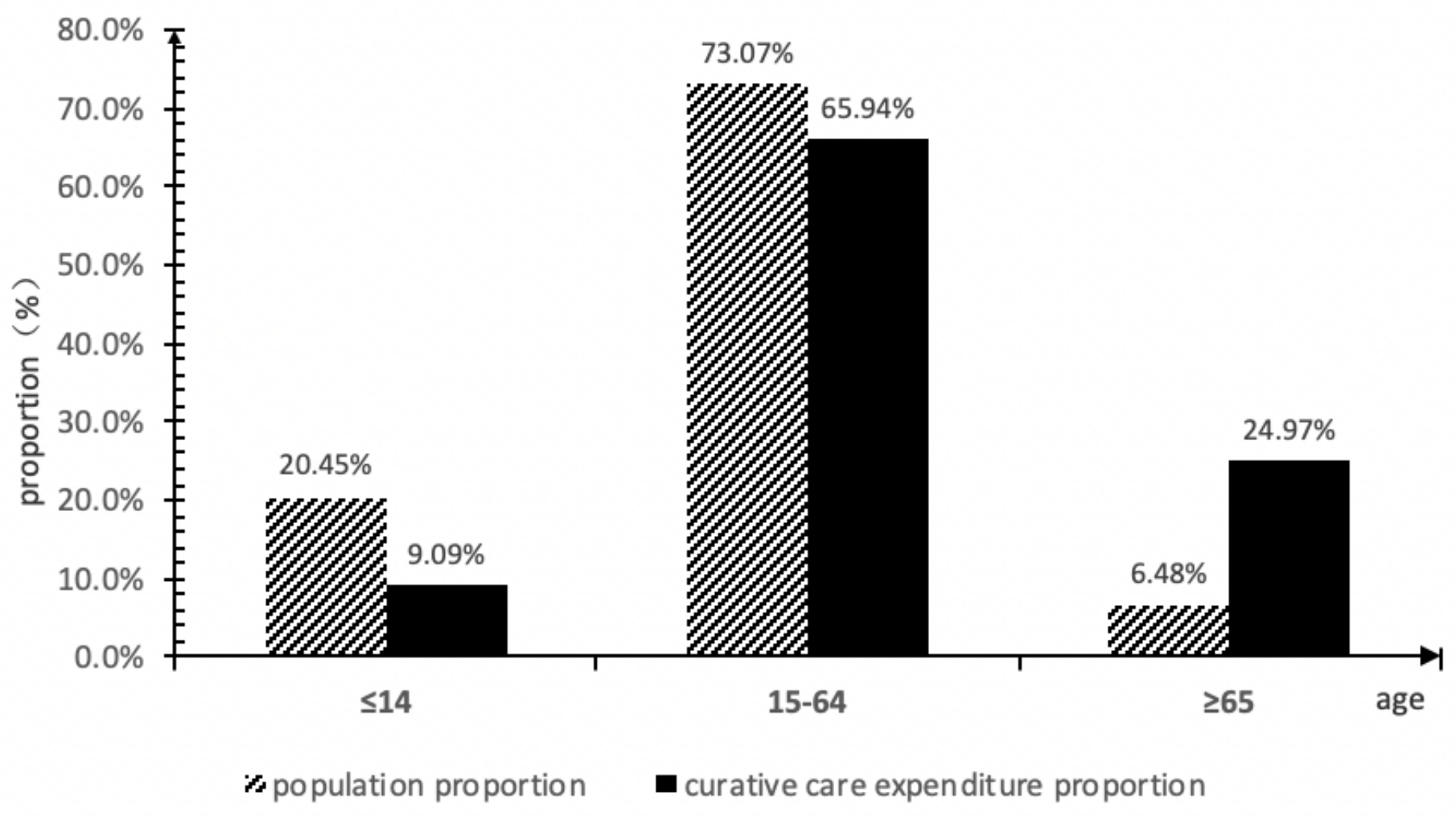




\section{Figure 1}

The proportion of the population and CCE accounting for age groups 